\title{
Study of the Three Phase Transformers under DC Bias and Suppression Measure
}

\author{
Dong Xia ${ }^{*}$ \\ School of Electrical Engineering and Automation \\ Qilu University of Technology \\ Jinan, China \\ dongxia6078@163.com \\ * Corresponding Author
}

\begin{abstract}
A novel transmission-line model (TLM) of the three phase transformers under direct current(DC) bias is built. In the model, the differential terms in the transformers circuit model are discretized. The saturation characteristic and the hysteresis effect of the core material are described on the basis of the Jiles-Atherton ferromagnetic hysteresis theory, and the eddy-current losses resulting from the flux paths through legs are replaced by an equivalent resistance. Furthermore, the copper losses and the leakage inductance of the primary and the secondary windings are also included. The nonlinear differential-algebraic equations can be obtained. At last, the Newton-Raphson technique is chosen to calculate the equations. The calculation results confirm the correctness and validity of the proposed the TLM for the three phase transformers under DC bias. At last, suppression measures of the $\mathrm{DC}$ bias are presented. The peak values of the exciting currents become smaller, and their distortion is partially reduced after taking the suppression measures.
\end{abstract}

Keywords-three phase transformers; DC bias; hysteretic effect; eddy-loss; saturation characteristic

\section{INTRODUCTION}

The DC bias phenomenon is abnormal for transformers. There are two reasons for generating DC bias, which one is geomagnetic induced current[1] (GIC, its frequency is lower then $0.01 \mathrm{~Hz}$ and can be considered to be DC); the other is DC transmission system (when DC transmission system uses earth as its current return path, great DC will flow in the earth, which will bring great ground potential differences in a large area around the DC grounding electrodes.) [2-3]. The DC bias can cause acute vibration, great noise, high temperature of the transformer, even make the protection misoperation. In addition, a lot of harmonics will be produced which can lower the power quality.

Some calculations and experiments have been done by a lot of researchers domestic and abroad. The main research contents included five parts as follows: (1) simulation analysis of exciting current [4-6]; (2) experiments research of the vibration, great noise, local high temperature of the transformer [7-8]; (3) research of leakage flux[9]; (4) measurement and simulation analysis of magnetization characteristic curve [10]; (5) research of restrain measures of DC bias [11-12]. Some achievements and progress have been achieved at above mentioned aspects.

This paper proposes a novel transmission-line model (TLM) of three phase transformers under DC bias. The differential terms of the transformers are replaced in TLM by the discrete transform. In the model, $B-H$ curve is defined on the basis of the Jiles-Atherton ferromagnetic hysteresis theory; eddy-current losses are replaced by an equivalent resistance. Furthermore, the copper losses and leakage inductance of the primary and the secondary windings are also included. The Newton-Raphson technique is chosen for its efficiency and stability to calculate the hysteretic behavior and exciting currents.

\section{TLM OF THREE PHASE TRANSFORMERS}

The equivalent electric circuit of the three phase transformers under DC bias is shown as Fig.1. Where $V_{\text {sa }}$, $V_{\mathrm{sb}}$ and $V_{\mathrm{sc}}$ are the three-phase AC voltages. $R_{\text {cua }}, R_{\text {cub }}, R_{\text {cuc }}$, $R_{\text {cuA }}, R_{\text {cuB }}, R_{\text {cuC }}$ are the resistances of the primary and the secondary windings respectively. $e_{\mathrm{a}}, e_{\mathrm{b}}, e_{\mathrm{c}}, e_{\mathrm{A}}, e_{\mathrm{B}}, e_{\mathrm{C}}$ are the induced electromotive forces of the primary and the secondary windings respectively. $i_{\mathrm{a}}, i_{\mathrm{b}}, i_{\mathrm{c}}, i_{\mathrm{A}}, i_{\mathrm{B}}, i_{\mathrm{C}}$ are currents of the primary and the second windings respectively. $L_{\mathrm{Las}}, L_{\mathrm{Lbs}}$ and $L_{\mathrm{Lcs}}$ are the equivalent leakage magnetic inductions which couple with the primary windings. $L_{\mathrm{LAS}}, L_{\mathrm{LBS}}$ and $L_{\mathrm{LCS}}$ are the equivalent leakage magnetic inductions which couple with the secondary windings. $R_{\mathrm{LA}}, R_{\mathrm{LB}}$ and $R_{\mathrm{LC}}$ are load resistances. $L_{\mathrm{LA}}, L_{\mathrm{LB}}$ and $L_{\mathrm{LC}}$ are load inductances. $\mathrm{M}$ is the mutual inductance of the first windings and the second windings. $U_{0}$ is the DC voltage.

The eddy-current losses of the transformers iron core are replaced by adding single-turn winding loaded by an

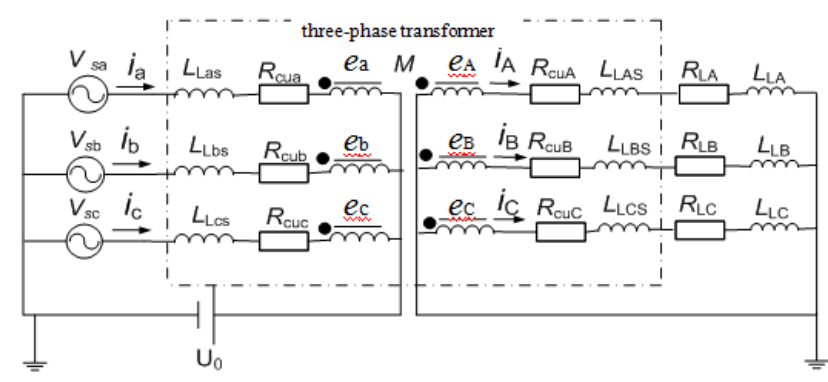

Figure 1. Electric circuit of the three phase transformers under DC bias 
equivalent resistance $R_{\mathrm{e}}$ given by[13]:

$$
R_{e}=\frac{12 A}{t^{2} \sigma_{f e} V_{c} K_{f e}}
$$

Where $A$ is the area of the iron core, $K_{f e}$ is the lamination factor of the iron core, $V_{\mathrm{c}}$ is the volume of the iron core, $\sigma_{f e}$ is the iron core conductivity, $t$ is the lamination thickness.

The TLM of three phase transformers[14] under DC bias is given as Fig. 2 and the equations can be derived as (2).
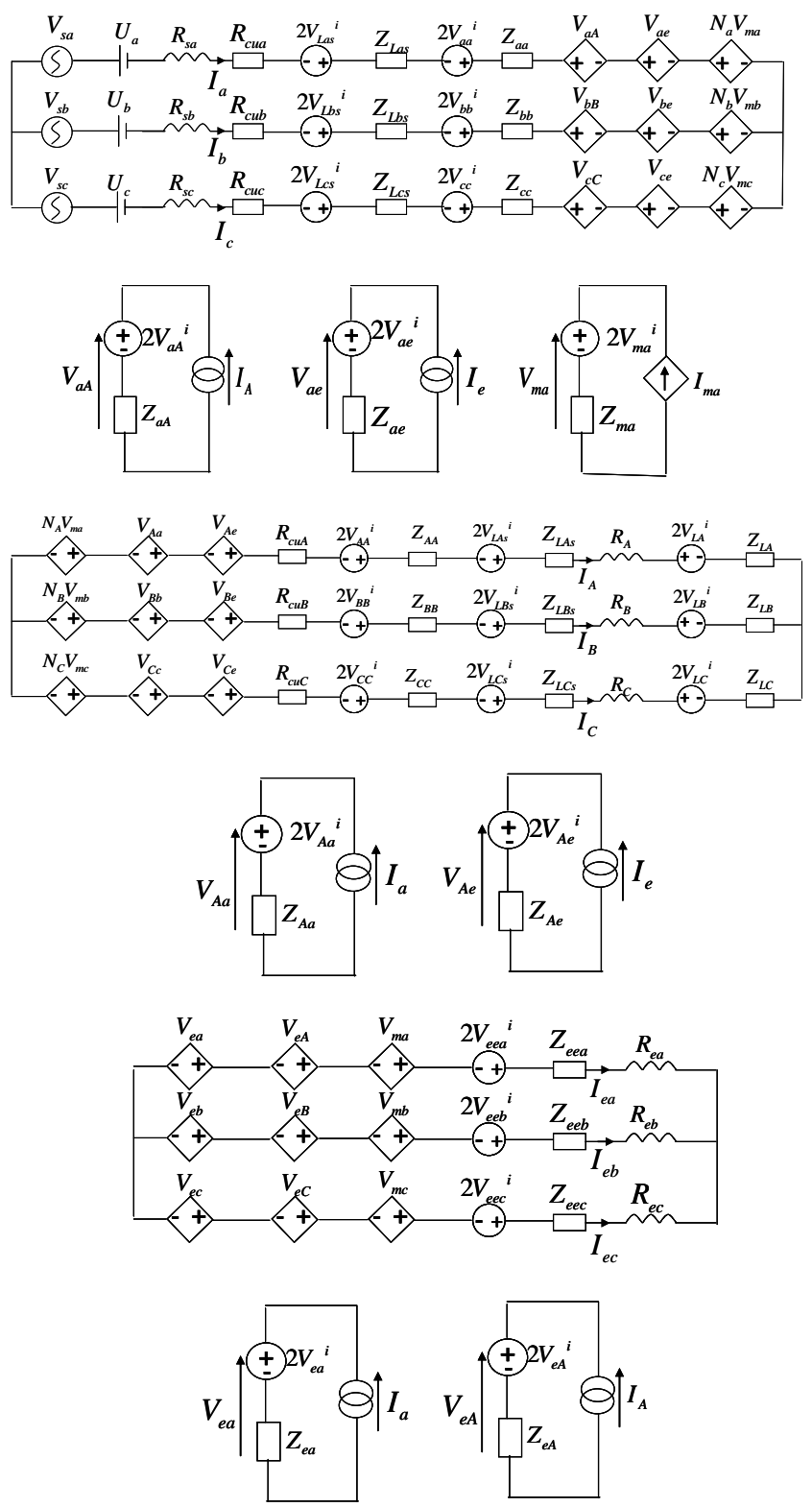

Figure 2. The TLM model of three-phase transformer

$V_{s p}+U_{p}=R_{s p} I_{p}+R_{c u p} I_{p}+L_{L p s} \frac{d I_{p}}{d t}+L_{p p} \frac{d I_{p}}{d t}+L_{p P} \frac{d I_{P}}{d t}+L_{p e} \frac{d I_{e}}{d t}+N_{p} L_{m} \frac{d I_{m}}{d t}$

$0=R_{\text {cuP }} I_{P}+L_{P P} \frac{d I_{P}}{d t}+L_{L P s} \frac{d I_{P}}{d t}+L_{P_{p}} \frac{d I_{p}}{d t}+L_{P e} \frac{d I_{e}}{d t}+N_{P} L_{m} \frac{d I_{m}}{d t}+R_{L P} I_{P}+L_{L P} \frac{d I_{P}}{d t}$ $0=L_{e e p} \frac{d I_{e}}{d t}+L_{e p} \frac{d I_{p}}{d t}+L_{e P} \frac{d I_{P}}{d t}+L_{m} \frac{d I_{m}}{d t}+R_{e p} I_{e p}$
Where the small letter subscripts represent the primary side parameters, the capital letter subscript represent the secondary side ones, and the letter $e$ subscript represents the additive winding. $V_{\mathrm{sp}}$ is AC voltage, $U_{\mathrm{p}}$ is DC voltage; $R_{\mathrm{sp}}$ are the internal resistances of the sources, $R_{\mathrm{cu}}$ are the resistances of the windings, $R_{\mathrm{LP}}$ are the resistances of the loads; $I_{\mathrm{p}}, I_{\mathrm{P}}$ and $I_{\mathrm{ep}}$ are the currents of the winding; $N_{\mathrm{p}}$ and $N_{\mathrm{P}}$ are the primary and the secondary winding turns; $L_{\mathrm{Ls}}$ are the leakage inductances; $L_{\mathrm{pp}}, L_{\mathrm{PP}}$ and $L_{\text {eep }}$ are the selfinductances, $L_{\mathrm{pP}}, L_{\mathrm{Pp}}, L_{\mathrm{pe}}, \mathrm{L}_{\mathrm{Pe}}, L_{\mathrm{ep}}$ and $L_{\mathrm{eP}}$ are the mutual inductances, $L_{\mathrm{LP}}$ are the inductances of the loads.

$$
\begin{aligned}
& L_{p p}=\mu_{0} N_{p}^{2} A / l, L_{P P}=\mu_{0} N_{P}^{2} A / l, L_{e e p}=\mu_{0} A / l, \\
& L_{p P}=L_{P p}=\mu_{0} N_{P} N_{p} A / l, L_{e p}=L_{p e}=\mu_{0} N_{p} A / l \\
& L_{e P}=L_{P e}=\mu_{0} N_{P} A / l, Z_{k}=2 L_{k} / d t .
\end{aligned}
$$

\section{JILES-ATHERTON THEORY}

The magnetization is split into two parts, the anhysteretic magnetization and the irreversible magnetization. In normalized form, this is expressed by

$$
I_{m}=\beta_{c} I_{a n}+\left(1-\beta_{c}\right) I_{i r r}
$$

Where $\beta_{c}$ is the weighting coefficient with $0 \leq \beta_{c} \leq 1$. The anhysteretic magnetization dependence is given by a modified Langevin function.

$$
I_{a n}=I_{s}\left[\operatorname{coth}\left(\frac{I_{h}+\alpha I_{m}}{I_{a}}\right)-\frac{I_{a}}{I_{h}+\alpha I_{m}}\right]
$$

Where $I_{\mathrm{s}}$ is the normalized saturation magnetization, $\alpha$ is the interdomain coupling coefficient, and $I_{\mathrm{a}}$ is the normalized anhysteretic magnetization form factor. The derivative of the normalized irreversible magnetization is

$$
\frac{d I_{i r r}}{d I_{h}}=\frac{\delta_{M}\left(I_{a n}-I_{i r r}\right)}{\delta I_{c}-\alpha\left(I_{a n}-I_{i r r}\right)}
$$

Where the migration flag $\delta_{M}$ is given by

$$
\delta_{M}=\left\{\begin{array}{l}
1, \text { if } \frac{d I_{h}}{d t}>0 \text { and }_{a n}>I_{\text {irr }} \\
1, \text { if } \frac{d I_{h}}{d t}<0 \text { and } I_{a n}<I_{\text {irr }} \\
0, \text { otherwise. }
\end{array}\right\}
$$

\section{CAlCUlation AND Simulation Result}

\section{A. Solution of the Transformer Model}

The Newton-Raphson technique is chosen to calculate the model.

$$
f=\left[\begin{array}{l}
f_{1} \\
f_{2} \\
f_{3}
\end{array}\right]_{9 \times 1}
$$

Where $f_{1}=\left[\begin{array}{l}f_{11} \\ f_{12} \\ f_{13}\end{array}\right]_{3 \times 1}, f_{2}=\left[\begin{array}{l}f_{21} \\ f_{22} \\ f_{23}\end{array}\right]_{3 \times 1}, f_{3}=\left[\begin{array}{l}f_{31} \\ f_{32} \\ f_{33}\end{array}\right]_{3 \times 1}$ 


$$
\begin{aligned}
& f_{11}=\left(R_{\text {sa }}+R_{\text {cua }}+Z_{a a}+Z_{\text {Las }}\right) I_{a}+Z_{a A} I_{A}+Z_{a e} I_{e}+N_{a} Z_{m a} I_{m a}-V_{s a}-U_{a}+2\left(V_{a a}{ }^{i}+V_{L a s}{ }^{i}+V_{a A}{ }^{i}+V_{a e}{ }^{i}+N_{a} V_{m a}{ }^{i}\right) \\
& f_{21}=\left(R_{A}+R_{c u A}+Z_{A A}+Z_{L A s}+Z_{L A}\right) I_{A}+Z_{A a} I_{a}+Z_{A e} I_{e}+N_{A} Z_{m a} I_{m a}+2\left(V_{A A}{ }^{i}+V_{L A s}{ }^{i}+V_{A a}{ }^{i}+V_{A e}{ }^{i}+N_{A} V_{m a}{ }^{i}\right) \\
& f_{31}=\left(R_{e a}+Z_{e e a}\right) I_{e}+Z_{e a} i_{a}+Z_{e A} I_{A}+Z_{m} I_{m}+2\left(V_{e e a}+V_{e a}{ }^{i}+V_{e A}{ }^{i}+V_{m}{ }^{i}\right)
\end{aligned}
$$

$f_{12}$ and $f_{13}, f_{22}$ and $f_{23}, f_{32}$ and $f_{33}$ are expressions of $\mathrm{b}$ and $\mathrm{c}$ phase.

Jacobean matrix is

$$
J=\left[\begin{array}{lll}
J_{11} & J_{12} & J_{13} \\
J_{21} & J_{22} & J_{23} \\
J_{31} & J_{32} & J_{33}
\end{array}\right]_{9 \times 9}
$$

Then the current can be solved.

$$
\left[\begin{array}{c}
I_{a} \\
I_{b} \\
I_{c} \\
I_{A} \\
I_{B} \\
I_{C} \\
I_{e a} \\
I_{e b} \\
I_{e c}
\end{array}\right]_{k+1}\left[\begin{array}{c}
I_{a} \\
I_{b} \\
I_{c} \\
I_{A} \\
I_{B} \\
I_{C} \\
I_{e a} \\
I_{e b} \\
I_{e c}
\end{array}\right]_{k}\left[\begin{array}{l}
f_{11}^{-1} \\
k \\
f_{12} \\
f_{13} \\
f_{21} \\
f_{22} \\
f_{23} \\
f_{31} \\
f_{32} \\
f_{33}
\end{array}\right]_{k}
$$

Where $k$ is the iteration number.

\section{B. Simulation Results}

To demonstrate the modeling procedure, a 100KVA, $10 \mathrm{KV} / 400 \mathrm{~V}$ three phase two-winding transformers was modeled using MATLAB soft.

Fig.3 4 show the simulated $B-H$ curve without DC bias and with $5 \mathrm{~V}$ DC voltage respectively. It can be seen that the curves are not symmetrical at the positive and negative $\mathrm{Y}$ axis any more with DC bias. The three phase transformers tends to saturate at the positive half of $\mathrm{Y}$ axis.

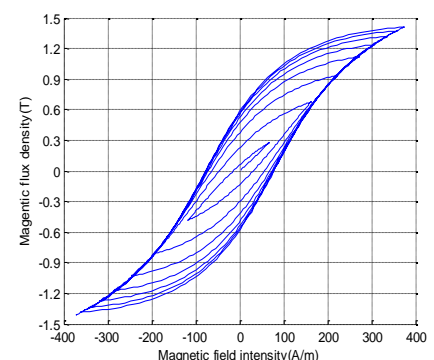

(a) a phase

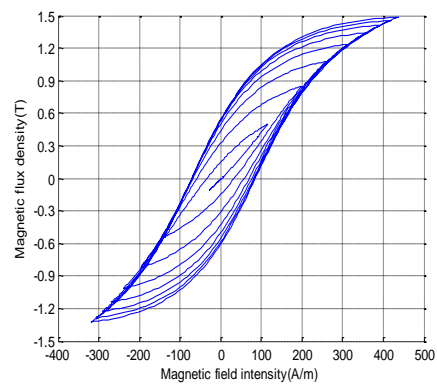

(b) b phase

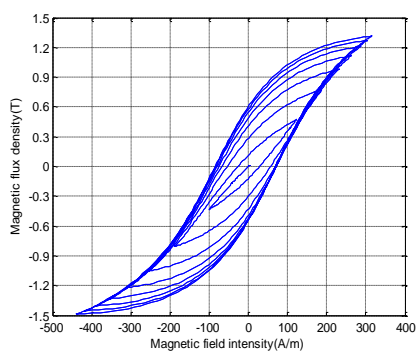

(c) c phase

Figure 3. $B-H$ curve of the three-phase transformer without DC voltage

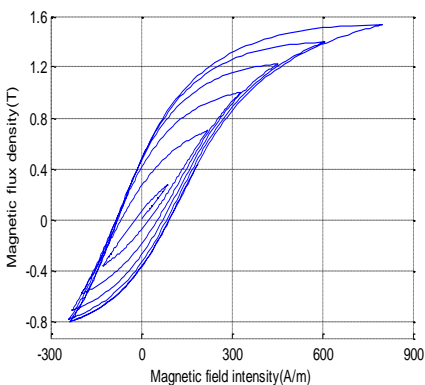

(a) a phase

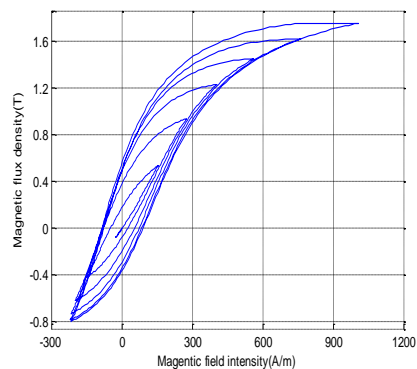

(b) b phase

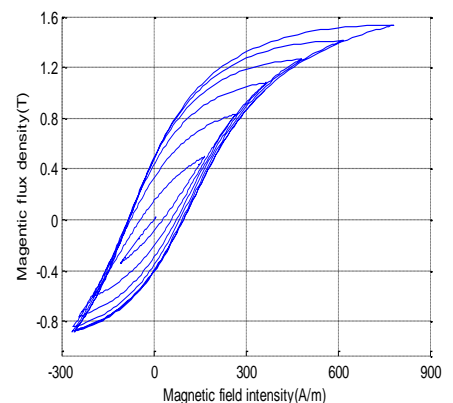

(c) c phase

Figure 4. $B-H$ curve of the three-phase transformer with $5 \mathrm{~V}$ DC voltage

Fig.5 6 are the exciting current simulated waveforms without DC bias and with $5 \mathrm{~V} \mathrm{DC}$ voltage respectively. It is obvious that the exciting currents waveforms get distorted with DC bias, and the larger the DC voltage is, the more seriously the exciting current waveforms distorted. The waveforms shift up and they are no longer symmetrical, which indicates that there are even harmonics occurred. 


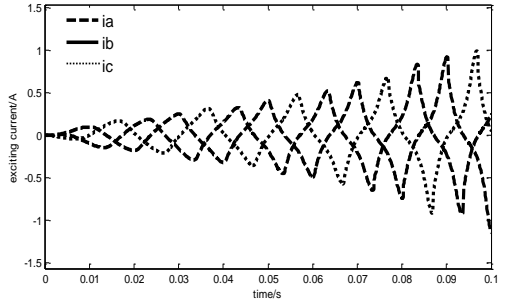

Figure 5. Exciting current waveform without DC voltage

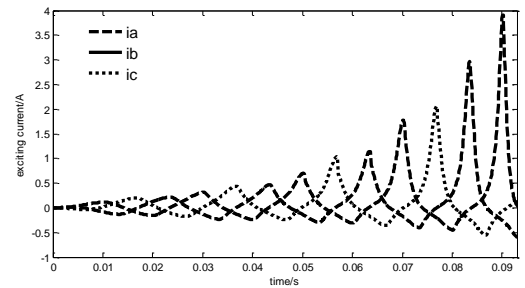

Figure 6. Exciting current waveform without 5V DC voltage

\section{SUPPRESSION MEASURE OF THE DC BIAS}

There are three main measures to restrain DC bias. They are (1) putting a resistor in series between the transformers neutral and ground, (2) putting a capacitor in series between the transformers neutral and ground, (3) putting a reverse DC injection at the neutral of the transformers.

Fig.7 is the exciting current simulated waveforms with $5 \mathrm{~V}$ DC voltage after applying suppression measures. It can be seen that the peak values of the exciting currents are smaller, and their waveforms distortion was partially reduced.

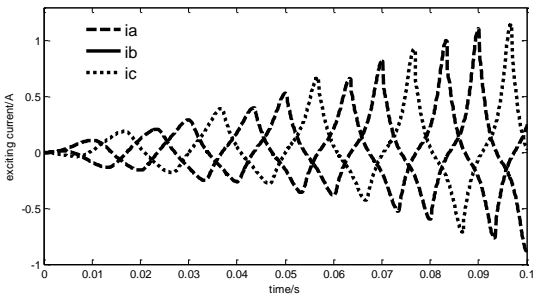

(a) putting a resistor

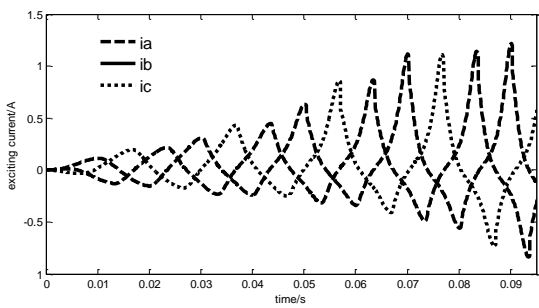

(b) putting a capacitor

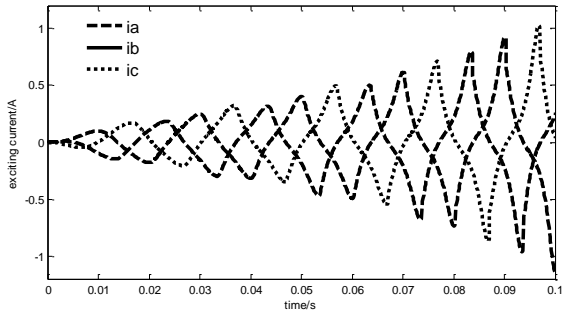

(c) putting a reverse dc injection

Figure 7. Exciting current waveforms with 5V DC voltage after applying suppression measure

\section{CONCLUSIONS}

1) The $B-H$ curves are not symmetrical at positive and negative $\mathrm{Y}$ axis any more under DC bias. The three phase transformers tends to saturate at the positive half of $Y$ axis.

2) The exciting current waveforms get distorted under DC bias, and the larger the DC voltage is, the more seriously the exciting current waveforms distorted.

3) The peak values of the exciting currents are smaller, and their waveforms distortion was partially reduced after applying suppression measure.

\section{REFERENCES}

[1] Cui Mingde, Liu Chunming, Liu Lianguang. Assessment of the influence caused by solar storm on sichuan power grid rated 500 kV[J]. High Voltage Engineering, 2010, 36(11):2849-2855.

[2] M.A.S. Masoum, P.S. Moses, Impact of balanced and unbalanced direct current bias on harmonic distortion generated by asymmetric three--phase three--leg transformers[J]. IET Electr. Power Appl. 2010,4(7):507-515.

[3] Yang Yongming, Liu Xingmou, Chen Tao, et al. Impact of soil structure adjacent to ground electrodes of UHVDC power transmission lines on DC bias of power transformers[J]. Power System Technology, 2012, 36(7):26-32.

[4] Li Xiaoping, Wen Xishan. DC Bias Computation Study on Threephase Five Limbs Transformer[J]. Proceedings of the CSEE 2010,30(1):127-131

[5] Zhao Xiaojun, Zhang Xiaoxin, Li Huiqi, et al. Frequency Domain Coupled Model between Magnetic and Electric Circuits of DC Biased Transformers by Harmonic Balance Method[J]. Transactions of China Electrotechnical Society. 2014,29(9):211218.

[6] Zhao Xiaojun, Li Lin, Cheng Zhiguang. et a1. Analysis of the DC bias phenomenon in transformers based on harmonic-balanced finite element method[J]. Proceedings of the CSEE, 2010,30(21) : 103-108.

[7] Wang Jin-gang, Mao Kai, Duan $\mathrm{Xu}$, et al. Simulation and test of transformer vibration under DC bias[J]. Electric Machines and Control, 2015,19(1):58-67.

[8] Zhao Zhigang, Liu Fuguil, Cheng Zhiguang, et al. Eddy current Loss of Copper Shielding Under DC--biased Condition in HVDC [J]. High Voltage Engineering. 2011,37(4):990-995.

[9] Guo Jie, Huang Hai, Tang Xin, et al. Analysis on $500 \mathrm{kV}$ Power Transformer Vibration Under DC M agnetic Biasing[J]. Power System Technology, 2012,36(3):70-75.

[10] Zhao Z, Liu F, Cheng Z. Measurements and calculation of core-based B-H curve and magnetizing current in DC-biased transformers[J], IEEE Transactions on Applied Super conductivity, 2010, 20(3):1131-1134

[11] Zhu Yiying, Jiang Weiping, Zeng Zhaohua, et al. Studyiing on Measures of Restraining DC current Through Transforme Neutrals[J]. Proceedings of the CSEE,2005,25(13):1-7.

[12] Huang FuCheng, Ruan Jiangjun, Zhang Yu, et al. DC Magnetic Bias Induced Current Effects on Transformer and Restricting Methods[J]. High Voltage Engineering,2006,32(9):117-120.

[13] Lu Changbai, Zhu Yinghao. Theory and calculation of power transformer[M]. 2006: LiaoNing Science and Technology Press.

[14] D.W.P Thomas, J. Paul, O.Ozgonenel, C. Christopoulos: TimeDomain Simulation of Nonlinear Transformers Displaying Hysteresis[J]. IEEE Transaction on Magnetics, 2006, 42(7) : 1820-1827. 